\title{
The structure of the $A B C$ transporter PsaBC shows that bacterial manganese import is achieved by unique architectural features that are conserved across the kingdoms of life.
}

\section{Stephanie L. Neville ${ }^{1}$, Jennie Sjöhamn ${ }^{2}$, Jacinta A. Watts ${ }^{1}$, Hugo MacDermott-Opeskin ${ }^{3}$, Stephen J. Fairweather ${ }^{3}$, Katherine Ganio ${ }^{1}$, Alex Carey Hulyer ${ }^{1}$, Andrew J. Hayes ${ }^{1}$, Aaron P. McGrath ${ }^{1}$, Tess. R. Malcolm ${ }^{1}$, Mark R. Davies ${ }^{1}$, Norimichi Nomura ${ }^{4}$, Iwata So ${ }^{4}$, Megan L. O’Mara ${ }^{3}$, Christopher A. McDevitt ${ }^{1}$, Megan J. Maher ${ }^{1}$}

\author{
${ }^{1}$ The University of Melbourne, Parkville, Australia; \\ ${ }^{2}$ University of Gothenburg, Gothenburg, Sweden.; \\ ${ }^{3}$ Australian National University, Canberra, Australia; \\ ${ }^{4}$ Kyoto University, Kyoto, Japan \\ megan.maher@unimelb.edu.au
}

Metal ions are essential for all forms of life. In prokaryotes, ATP-binding cassette (ABC) permeases serve as the primary import pathway for many micronutrients including the first-row transition metal manganese. However, the structural features of ionic metal transporting $\mathrm{ABC}$ permeases have remained undefined. This presentation will describe the crystal structure of the manganese transporter PsaBC from Streptococcus pneumoniae in an open-inward conformation. The Type II transporter has a tightly closed transmembrane channel due to 'extracellular gating' residues that prevent water permeation or ion reflux. Below these residues, the channel contains a hitherto unreported metal coordination site, which is essential for manganese translocation. These structural features are highly conserved in metal-specific ABC transporters and are represented throughout the kingdoms of life. Collectively, our results define the structure of $\mathrm{PsaBC}$ and reveal the features required for divalent cation transport.

Keywords: membrane protein, X-ray crystallography, Streptococcus pneumoniae, manganese 